\title{
Suicide Behaviors and Health-Related Quality of Life: Results from the Korean Community Health Survey of 393,073 Adults
}

\author{
Jin-young Min ${ }^{1}$ and Kyoung-bok Min ${ }^{2 *}$ \\ ${ }^{1}$ Institute of Health and Environment, School of Public Health, Seoul National University \\ ${ }^{2}$ Department of Occupational and Environmental Medicine, Ajou University School of Medicine, Suwon, Republic of Korea
}

*Corresponding author: Kyoung-Bok Min, Department of Occupational and Environmental Medicine, Ajou University School of Medicine, San 5, Wonchon-dong, Yeongtong-gu, Suwon, 443-721, Republic of Korea, Tel: +82-31-219-5278; Fax: +82-31-241-1697; E-mail: mink1999@ajou.ac.kr

Received Date: November 11, 2014, Accepted Date: November 25, 2014, Published Date: December 3, 2014

Copyright: (c) 2015, Jin-young Min et al., This is an open-access article distributed under the terms of the Creative Commons Attribution License, which permits unrestricted use, distribution, and reproduction in any medium, provided the original author and source are credited.

\begin{abstract}
Background: Suicide is a major public health concern worldwide, but it is largely preventable. Although previous studies have suggested quality of life as a predictor of suicide risk, knowledge about this topic is limited by the small number of available subjects and insufficient information. The aim of this study is to investigate the association between the EuroQoL 5 dimension (EQ-5D) measurement and the risk of suicidal behaviors among adults in a representative sample of Korea.
\end{abstract}

Subjects and Methods: The data were obtained from the 2008 and 2009 Korean Community Health Survey. A total of 393,073 participants older than 20 years were included in this study. These participants had no missing information on questionnaires about the EQ-5D measures, suicide behaviors, and other covariates. Of these subjects, 37,689 experienced only suicide ideation and 1,910 subjects experienced both suicide ideation and suicide attempts.

Results: We found that participants who experienced suicide ideation and/or suicide attempts had significantly lower quality of life than those who had not experienced such behaviors. After adjusting for confounding variables, the estimate of EQ-5D score was lowest in subjects with both suicide ideation and suicide attempts, followed by subjects with only ideation and then subjects without suicide behaviors.

Conclusions: Considering that suicide ideation and suicide attempts are important factors in the likelihood of eventual suicide, assessing quality of life may be beneficial for identifying adults at high-risk of suicide and for reducing suicide-related risks at the population level.

Keywords: Suicide ideation; Suicide attempt; Quality of life; Community health survey

\section{Introduction}

As one of the leading causes of death worldwide, suicide is a major concern for public health authorities. The World Health Organization has estimated that almost one million people commit suicide annually, which is an average of one suicide death every 40 seconds [1]. Suicide rates are traditionally highest for the elderly people; however, the rates are rising for adolescents and young adults, which make this an important problem for all age groups [2].

Numerous factors lead to suicide behaviors, including, importantly, suicide ideation and suicide attempts. Known risk factors include previous self-harming actions, a family history of suicide, personality characteristics (e.g, impulsivity and aggression), socioeconomic status, and misuse of alcohol or drugs [2,3]. Psychiatric disorders (e.g., depression, misuse of alcohol or drugs, schizophrenia, and bipolar disorder)and poor physical health and disabilities are also known to be powerful risk factors [2,3]. However, the implications of each individual factor likely to be minor; because each factor accounts for only a small fraction of the variance in individual suicide risk and has insufficient risk specificity [4]. Because suicide is largely preventable, identifying individuals at high-risk for suicide is an important component of an effective approach to suicide prevention, but this task remains challenging. However, comprehensive risk-factor assessment might be effective in suicide prevention at the population level.

Health-related quality of life (HRQOL) indices are multidimensional and comprehensive measures of a subject's perceptions of his or her physical, mental, emotional, and social functioning and are widely recognized as independent predictors of morbidity and mortality [5]. Previous studies have revealed a significant association between HRQOL and suicide behaviors [6-12]. Although the assessment of HRQOL provides valuable insight into the risk of suicide, research knowledge is limited by the small number of subjects with suicidal behaviors, the generally older population of subjects with suicidal behaviors, patients with a specific disease, and/or insufficient adjustment for confounding variables.

In Korea, suicide is a major social issue; the suicide rate has increased dramatically over recent decades, growing from 13.0 per 100,000 people in 1997 to 33.3 per 100,000 in 2011 [13,14]. This rate is the highest among countries belonging to the Organization for Economic Cooperation and Development [14]. Thus, the magnitude of South Korea's suicide problem includes many issues in addition to suicide ideation and/or suicide attempts. Given that previous studies 
Page 2 of 7

on suicide risk have limitations due to small number of subjects with suicide behaviors [2], which make them vulnerable to error, the results of the present study appear to be relatively stable.

In this study, we sought to investigate the association between HRQOL and the risk of suicidal behaviors among adults in a representative sample from Korea. We hypothesized that adults who experienced suicidal behaviors would be more susceptible to low HRQOL than those with no suicidal behaviors. Using a large and representative data sample from Korea, we employed the EuroQoL 5 Dimension (EQ-5D) as the index for measuring HRQOL and included suicide behaviors, particularly with respect to suicidal ideation alone, suicidal ideation coupled with attempted suicide, and the absence of suicidal behaviors.

\section{Materials and Methods}

\section{Study population}

We used data from the 2008 and 2009 Korean Community Health Survey (KCHS), conducted by the Korea Centers for Disease Control and Prevention. The KCHS is a cross-sectional study that began in 2008 with the goals of gathering reliable health-related data at the community level in different geographical regions and providing evidence-based public health programs and services. Study participants were recruited from public health centers in subcommunities using probability proportions to size systematic sampling and root proportional allocation. The survey was conducted annually over the September-November period and gathered information through fact-to-face, paper-assisted personal interviews between trained interviewers and respondents. The information included socio-demographic information, health behaviors (i.e., smoking and alcohol consumption), self-reported health status, health system utilization, and quality of life. A total of 450,973 participants (220,258 in 2008 and 230,715 in 2009) were included in the study [15].

Of the 450,973 participants, we excluded 1221 subjects who did not respond or incompletely responded to the EQ-5D and questions about suicide behaviors. Additionally, 56679 subjects with missing variables of interest were excluded, which resulted in the inclusion of a total of 393,073 participants $(87.2 \%)$ in this study.

\section{Suicidal behaviors}

Individuals' suicidal behaviors were obtained through self-reporting report of suicide ideation and suicide attempts. Suicide ideation was based on a question about whether the respondent had thought about dying in the past 12 months. Suicidal attempts were based on a question about whether the respondent had attempted suicide in the past 12 months. The responses were coded as dichotomous variables ("yes" or "no answer").

\section{EQ-5D measurement}

HRQOL was measured by the EQ-5D, which was developed by the EuroQol Group[16]. The EQ-5D is an instrument that consists of five dimensions of the current health status: mobility (M), self-care (SC), usual activities (UA), pain/discomfort (PD), and anxiety/depression (AD). Each dimension has the following three possible response: no problem (1); some problems (2); and severe problems (3).

Using a combination of these dimensions, a single summary score was calculated for the EQ-5D. Scores on the EQ-5D ranged from
-0.171 to 1 , where 1 indicates no problems in any of the five dimensions, zero indicates death, and a negative score indicates a health status worse than death. The Korean version of the EQ-5D was validated by the Korean Centers for Disease Control and Prevention [17].

\section{Variables}

Variables of interest in the association between quality of life and suicidal behaviors were determined based on evidence in the previous literature. As part of the KCHS, respondents provided information about their age (20-39, 40-59, or $\geq 60$ years), gender, marital status (married, divorced or widowed, or never married), monthly income (1Q: $\leq 1000$ thousand won, 2Q: 1000-2000 thousand won, 3Q: 2000-3300 thousand won, 4Q: $\geq 3301$ thousand won), and education (less than high school, high school, college or higher). Job categories were white-collar (managerial, professional, or clerical), pink-collar (services or sales), blue-collar (manual), military, or no economic activity. Health behaviors included smoking status (current, former, or never) and alcohol consumption (drinker or non-drinker).

Health status was divided into two segments: self-estimated health status (very good, good, moderate, bad, or very bad) and previous physician-diagnosed diseases (i.e., hypertension, diabetes mellitus, dyslipidemia, stroke, myocardial infarction, or angina). Feelings of depression were identified through a question asking the respondent whether he/she had experienced feelings of depression in the past 12 months ("yes" or "no answer").

\section{Statistical analysis}

All analyses of suicidal behaviors were conducted separately in the following categories: subjects without suicidal behaviors ('no suicidal behaviors'), subjects with suicide ideation ('suicide ideation'), and subjects with both suicide ideation and suicide attempts ('suicide ideation and suicide attempts'). The EQ-5D index was calculated based on the recommendation of the Korean Centers for Disease Control and Prevention [17]. The formula to convert scores for the five dimensions is described in the following:

$\mathrm{EQ}-5 \mathrm{D}$ index $=1-(0.05+0.096 \times \mathrm{M} 2+0.418 \times \mathrm{M} 3+0.046 \times \mathrm{SC} 2+$ $0.136 \times \mathrm{SC} 3+0.051 \times \mathrm{UA} 2+0.208 \times \mathrm{UA} 3+0.037 \times \mathrm{PD} 2+0.151 \times \mathrm{PD} 3+$ $0.043 \times \mathrm{AD} 2+0.158 \times \mathrm{AD} 3+0.05 \times \mathrm{N} 3)$.

Where the numbers 2 and 3 indicate levels of severity for the five dimensions of the EQ-5D ('some problems' and 'severe problem', respectively). N3 is rescaled from 0 to 1 if at least one dimension at level 2 or 3 (some or severe problems) is 1; otherwise, N3 is set at 0 ).

Differences in quality of life according to suicidal behaviors were assessed by the mean of the EQ-5D scores and the frequency of subjects with 'some' or 'severe' problems in the five dimensions of the EQ-5D. A general linear regression model was conducted by treating the EQ-5D index as continuous variables. We estimated models by adjusting for covariates: Model I was adjusted for age, gender, and marital status; Model II was adjusted for Model I + income, education, job categories, smoking, and alcohol consumption; Model III was adjusted for Model II + self-estimated health status and histories of disease of hypertension, diabetes mellitus, dyslipidemia, stroke, myocardial infarction, and/or angina. Each model produced the adjusted estimates $(\beta)$ and standard error (SE) of the EQ-5D in subjects with either suicide ideation or with both suicide ideation and 
suicide attempts. Subjects without suicidal behaviors were designated as the reference group.

All of the analyses were performed using SAS 9.2 (SAS Institute, Cary, NC, USA), and the statistical significance level was set at $\alpha=.05$.

\section{Results}

Of the 393,073 participants, approximately $10 \%(n=39,599)$ of the subjects reported suicidal behaviors (37,689 subjects with only suicide ideation and 1,910 subjects with both suicide ideation and suicide attempts).

Table 1 shows the mean differences in the EQ-5D score between study participants with different demographic, health and suicidal behavior characteristics. The mean score on the EQ-5D decreased with age, and females had lower scores than males. Divorced/widowed subjects had a lower quality of life than participants in other marital status categories. As monthly income and educational level rose, quality of life increased significantly. Employed participants were more likely to have high EQ-5D scores than those who were unemployed. Current smokers or drinkers had better quality of life than those who were former/non smokers or non-drinkers. Participants who reported very good self-estimated health status had the highest score on the EQ-5D, and those who reported very poor self-estimated health status had the lowest scores. Regarding disease status, participants who had hypertension, diabetes, dyslipidemia, stroke, myocardial infarction, or angina were likely to have lower EQ-5D scores than those without such diseases. Participants who reported feelings of depression had lower quality of life than those without feelings of depression. Suicidal behaviors significantly reduced quality of life; subjects who had both suicide ideation and suicide attempts had the lowest EQ-5D scores, The next group that had experienced only suicide ideation also had low EQ-5D score. Subjects without suicidal behaviors scored the highest on the EQ-5D among the groupings. When the participants' ages were factored into the three groups (20-39 years, or young-aged adults; 40-59 years, or middleaged adults; and $\geq 60$ years, or older adults, the mean EQ-5D showed a similar pattern for suicidal behaviors (data not shown): subjects without suicide behaviors scored higher than subjects with only suicide ideation, who, in turn, scored higher than subjects with both suicide ideation and suicide attempts.

\begin{tabular}{|c|c|c|c|c|}
\hline & $\mathbf{n}$ & Mean & (SD) & $p$-value* \\
\hline \multicolumn{5}{|l|}{ Age (years) } \\
\hline $20 \sim 39$ & 118774 & 0.98 & -0.06 & $<.0001$ \\
\hline 40 59 & 152520 & 0.96 & -0.09 & \\
\hline $60 \sim$ & 121779 & 0.86 & -0.19 & \\
\hline \multicolumn{5}{|l|}{ Gender } \\
\hline Male & 181874 & 0.95 & -0.12 & $<.0001$ \\
\hline Female & 211199 & 0.92 & -0.14 & \\
\hline \multicolumn{5}{|l|}{ Marital status } \\
\hline Married & 283200 & 0.95 & -0.12 & $<.0001$ \\
\hline Divorced/Widowed & 55407 & 0.85 & -0.19 & \\
\hline Never Married & 54466 & 0.98 & -0.08 & \\
\hline \multicolumn{5}{|l|}{ Monthly Income (KRW) } \\
\hline $1 \mathrm{Q}(\sim 1000$ thousand $)$ & 114530 & 0.88 & -0.18 & $<.0001$ \\
\hline $2 Q(\sim 2000$ thousand $)$ & 96714 & 0.95 & -0.11 & \\
\hline $3 Q(\sim 3300$ thousand $)$ & 82694 & 0.97 & -0.09 & \\
\hline $4 \mathrm{Q}(3301 \sim$ thousand $)$ & 99135 & 0.97 & -0.08 & \\
\hline \multicolumn{5}{|l|}{ Education } \\
\hline Elementary or lower & 112717 & 0.86 & -0.18 & $<.0001$ \\
\hline Middle to High school & 166272 & 0.96 & -0.1 & \\
\hline College or higher & 114084 & 0.98 & -0.06 & \\
\hline \multicolumn{5}{|l|}{ Job Categories } \\
\hline White-collar & 71222 & 0.99 & -0.05 & $<.0001$ \\
\hline
\end{tabular}


Citation: Jin-Young Min Kyoung-bok Min (2015) Suicide Behaviors and Health-Related Quality of Life: Results from the Korean Community Health Survey of 393,073 Adults. J Psychiatry 18: 214. doi:10.4172/2378-5756.1000214

Page 4 of 7

\begin{tabular}{|c|c|c|c|c|}
\hline Pink-collar & 48694 & 0.97 & -0.07 & \\
\hline Blue-collar & 118415 & 0.95 & -0.09 & \\
\hline Military & 1864 & 0.99 & -0.05 & \\
\hline Unemployed & 152878 & 0.89 & -0.18 & \\
\hline \multicolumn{5}{|l|}{ Smoking } \\
\hline Current smoker & 93771 & 0.96 & -0.11 & $<.0001$ \\
\hline Former smoker & 54172 & 0.93 & -0.16 & \\
\hline Never-smoker & 245130 & 0.93 & -0.13 & \\
\hline \multicolumn{5}{|c|}{ Current alcohol consumption } \\
\hline Drinker & 214379 & 0.96 & -0.09 & $<.0001$ \\
\hline Non-drinker & 178694 & 0.91 & -0.17 & \\
\hline \multicolumn{5}{|c|}{ Self-estimated health status } \\
\hline Very good & 19845 & 0.99 & -0.05 & $<.0001$ \\
\hline Good & 142855 & 0.98 & -0.05 & \\
\hline Moderate & 145622 & 0.96 & -0.08 & \\
\hline Bad & 68456 & 0.85 & -0.16 & \\
\hline Very bad & 16295 & 0.64 & -0.29 & \\
\hline \multicolumn{5}{|l|}{ Hypertension } \\
\hline Yes & 77551 & 0.87 & -0.18 & $<.0001$ \\
\hline No & 315522 & 0.95 & -0.11 & \\
\hline \multicolumn{5}{|l|}{ Diabetes mellitus } \\
\hline Yes & 28191 & 0.86 & -0.2 & $<.0001$ \\
\hline No & 364882 & 0.94 & -0.13 & \\
\hline \multicolumn{5}{|l|}{ Dyslipidemia } \\
\hline Yes & 21712 & 0.9 & -0.15 & $<.0001$ \\
\hline No & 371361 & 0.94 & -0.13 & \\
\hline \multicolumn{5}{|l|}{ Stroke } \\
\hline Yes & 8236 & 0.69 & -0.3 & $<.0001$ \\
\hline No & 384837 & 0.94 & -0.12 & \\
\hline \multicolumn{5}{|c|}{ Myocardial infarction } \\
\hline Yes & 4478 & 0.82 & -0.22 & $<.0001$ \\
\hline No & 388595 & 0.94 & -0.13 & \\
\hline \multicolumn{5}{|l|}{ Angina } \\
\hline Yes & 5774 & 0.83 & -0.2 & $<.0001$ \\
\hline No & 387299 & 0.94 & -0.13 & \\
\hline Depressive feeling & & & & \\
\hline
\end{tabular}


Citation: Jin-Young Min Kyoung-bok Min (2015) Suicide Behaviors and Health-Related Quality of Life: Results from the Korean Community Health Survey of 393,073 Adults. J Psychiatry 18: 214. doi:10.4172/2378-5756.1000214

Page 5 of 7

\begin{tabular}{|l|l|l|l|l|}
\hline Yes & 31670 & 0.82 & -0.23 & $<.0001$ \\
\hline No & 361403 & 0.95 & -0.12 & \\
\hline Suicide behaviors & & & & \\
\hline No suicide behaviors & 353485 & 0.95 & -0.11 & $<.0001$ \\
\hline Suicide ideation & 37680 & 0.83 & -0.22 & \\
\hline Suicide ideation and attempts & 1908 & 0.78 & -0.23 & \\
\hline
\end{tabular}

Table 1: Mean (SD) differences of EQ-5D by study participants' characteristics (N=393,073)

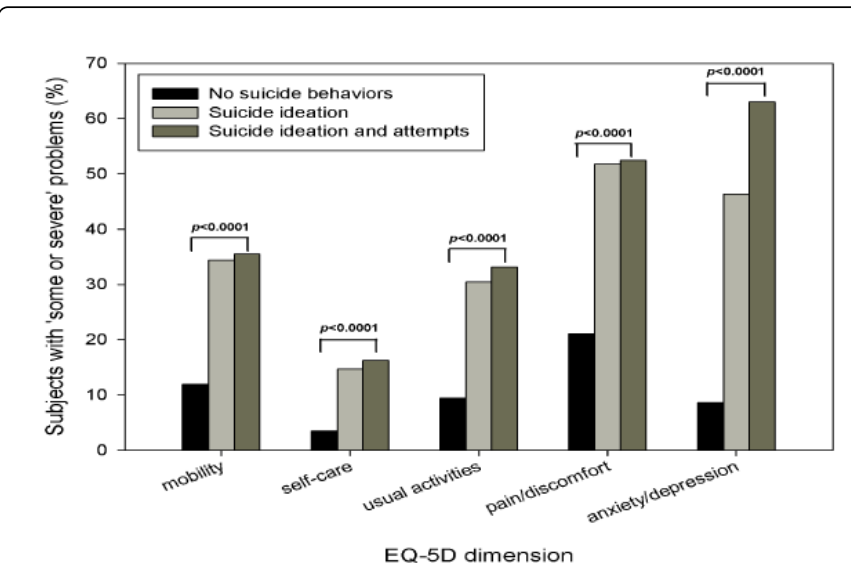

Figure 1: Percentages of subjects with 'some' or 'severe' problems in each EQ-5D dimension according to suicide behaviors.
Figure 1 presents the percentages of subjects with 'some' or 'severe' problems in each EQ-5D dimension categorized according to suicidal behaviors. Subjects with suicidal behaviors were substantially more likely to report problems with mobility, self-care, usual activities, pain/ discomfort, and anxiety/depression compared to subjects without suicidal behaviors. Within the group with suicidal behaviors, subjects with both suicide ideation and suicide attempts were more likely to have problems in each EQ-5D dimension than those with only suicide ideation.

Table 2 shows the adjusted estimates for the EQ-5D by suicidal behaviors in each age group. We observed a significant negative association of the EQ-5D with suicide behaviors. When adjusted for participants' socioeconomic characteristics and health behaviors (model 1 and model 2), compared to subjects without suicide behaviors (reference group), the estimates of the EQ-5D were significantly reduced in subjects with suicide ideation and/or suicide attempts. Additional adjustments for health status (self-rated health, hypertension, diabetes, dyslipidemia, stroke, MI, angina, and feelings of depression; model 3) resulted in an even more dramatic reduction in quality of life in subjects with suicide behaviors. The observed results were consistent across all adult age groups. In particular, the estimate reduction of older adults ( $\geq 60$ years) was greater than other adult groups $(-0.058$ for subjects with only suicide ideation and -0.064 for subjects with both suicide ideation and suicide attempts).

\begin{tabular}{|c|c|c|c|c|c|c|c|c|c|}
\hline & \multicolumn{3}{|c|}{ 20 39 years old } & \multicolumn{3}{|c|}{$40 \sim 59$ years old } & \multicolumn{3}{|c|}{$\geq 60$ years old } \\
\hline & Estimate & $(\mathrm{SE})$ & p-value & Estimate & (SE) & $\mathrm{p}$-value & Estimate & (SE) & $p$-value \\
\hline \multicolumn{10}{|c|}{ Model 1 (adjusted for age, gender, and marital status) } \\
\hline No suicide behaviors & \multicolumn{3}{|c|}{ Reference } & \multicolumn{3}{|c|}{ Reference } & \multicolumn{3}{|l|}{ Reference } \\
\hline Suicide ideation & -0.048 & $(0.001)$ & $<.0001$ & -0.077 & $(0.001)$ & $<.0001$ & -0.149 & $(0.001)$ & $<.0001$ \\
\hline Suicide ideation and attempts & -0.104 & $(0.003)$ & $<.0001$ & -0.152 & $(0.003)$ & $<.0001$ & -0.198 & $(0.007)$ & $<.0001$ \\
\hline \multicolumn{10}{|c|}{ Model 2 (adjusted for model $1+$ job, education, income, smoking, and drinking) } \\
\hline No suicide behaviors & \multicolumn{3}{|c|}{ Reference } & \multicolumn{3}{|c|}{ Reference } & \multicolumn{3}{|l|}{ Reference } \\
\hline Suicide ideation & -0.047 & $(0.001)$ & $<.0001$ & -0.072 & $(0.001)$ & $<.0001$ & -0.136 & $(0.001)$ & $<.0001$ \\
\hline Suicide ideation and attempts & -0.101 & $(0.003)$ & $<.0001$ & -0.141 & $(0.003)$ & $<.0001$ & -0.007 & $(0.007)$ & $<.0001$ \\
\hline
\end{tabular}




\begin{tabular}{|c|c|c|c|c|c|c|c|c|c|}
\hline \multirow{2}{*}{$\begin{array}{l}\text { No suicide behaviors } \\
\text { Suicide ideation }\end{array}$} & \multicolumn{3}{|c|}{ Reference } & \multicolumn{3}{|c|}{ Reference } & \multicolumn{3}{|c|}{ Reference } \\
\hline & -0.025 & $(0.001)$ & $<.0001$ & -0.036 & $(0.001)$ & $<.0001$ & -0.058 & $(0.001)$ & $<.0001$ \\
\hline Suicide ideation and attempts & -0.059 & $(0.002)$ & $<.0001$ & -0.074 & $(0.003)$ & $<.0001$ & -0.064 & $(0.006)$ & $<.0001$ \\
\hline
\end{tabular}

Table 2: Adjusted estimates (SE) of EQ-5D by suicide behavior for each age group

\section{Discussion}

We found that adults who experienced suicide ideation and/or suicide attempt had significantly lower scores for the EQ-5D than those who had not experienced such behaviors. This association was independent of other confounding variables, such as socioeconomic characteristics, physical health status, and feelings of depression, and was consistent across all adult age groups. Our study extends the existing evidence on the usefulness of quality of life as a predictor for suicide risk, and suggests that employing quality of life issues in suicide risk detection and reduction may be beneficial across all adultage groups.

Consistent with our findings, previous studies have suggested that reduced quality of life is significantly associated with suicide behaviors [6]. In a study of a representative sample of the South Australian population, Goldney et al (2001) investigated the HRQOL of people with suicide ideation. Of 14,456 respondents over the age of 15 years, 79 subjects $(2.6 \%)$ reported suicide ideation. Those with suicide ideation had significantly poorer quality of life measurements $(\mathrm{P}<0.001)$ than those without suicidal ideation [6]. A recent study suggested that the quality of life of older adults strongly affects suicide behaviors, finding that older adults with an EQ-5D index score $<0.7$ had a 9.1 and 3.4-times greater odds ratios for suicide ideation and suicide attempts than those with a relatively high index score of 1 or $0.8-1$, respectively [8]. In addition, suicidal risk in patients with schizophrenia was significantly associated with poor social and psychological quality of life, identifying important subgroups of the patients at high risk for suicide [10-12]. In patients with diabetes mellitus or bipolar disorder, lower HRQOL were significant contributors to suicidal behavior [7,9]. These findings support the need for quality of life assessments to identify people at risk for suicide behaviors with a view toward early intervention.

A particularly interesting finding of the current study is that quality of life, as measured by the EQ-5D, enables the potential detection of the severity of possible suicide behaviors. We observed that subjects who reported both suicide ideation and suicide attempts had the lowest score on the EQ-5D. The next lowest score was found for those who reported only suicide ideation without suicide attempts, and those who did not experience suicide ideation or suicide attempts had the highest quality of life. Although quality of life decreased with age, regardless of suicide behaviors, the pattern of a reduction in EQ-5D scores accompanied by an increase in the degree of suicidal behaviors was also common in all age groups. Because that suicide ideation is an important antecedent to suicide attempts and because these non-fatal suicide behaviors are relatively high-risk factors for eventual suicide [18-20], quality of life assessment deserves more attention for suicide prevention in community and medical care.

HRQOL reflects quality of life relative to one's physical, social, psychological, and spiritual factors. Although HRQOL involves a lack of conceptual clarity and measurement feasibility [21,22], epidemiological studies have widely recognized HRQOL measurement as a consideration of disease stage or treatment/intervention effects in patients and as a determinants of disparity in specific conditions (i.e., socioeconomic status, employment, and disease prevalence) in the general population[23-28]. As applied to current study, the EQ-5D applied to the current study is a valid generic instrument for health status that captures health in terms of mobility, self-care, usual activities, pain/discomfort, and anxiety/depression. It has been used in clinical and economic evaluations of health care and in population health surveys in many countries [29]. Whether the EQ-5D is a reasonable measure for screening the population for suicide risk remains unclear, but our study indicates that subjects who reported suicide behaviors were more susceptible to decreased quality of life compared to subjects who did not experience suicide behaviors. Thus, self-reporting quality of life may be an important factor in suicide behaviors.

This study is the first to show a strong association between impaired quality of life and suicidal behaviors among all adults using a large and representative sample dataset and events related to suicide behaviors. However, several limitations of this study should be noted in the interpretation of the findings. The study was based on a cross-sectional design; thus, we cannot definitely establish a causal relationship between quality of life and the risk of suicide behaviors. Nevertheless, previous studies provide evidence that subjects with poor quality of life are more likely to report suicide behaviors. The KCHS included only self-reported data, which are likely to involve recall bias for social desirability. In addition, the reliability of psychometric measures, including suicide behaviors and feelings of depression as applied to the KCHS was implicitly assumed rather than explicitly ascertained. Finally, due to the observational nature of this research, we cannot rule out the possibility of residual confounding effects by unmeasured confounders. For example, because suicide behaviors are complex and involve physical, mental, and social factors, there are many possible precipitating factors (i.e., family history of suicide, childhood environment, or abuse of drugs) for these behaviors that we cannot measure.

\section{Conclusions}

We found that suicide behaviors were significantly associated with low quality of life as assessed by the EQ-5D among younger, middleaged, and older adults. Given that suicide ideation and/or suicide attempts are important factors in eventual suicide, assessing quality of life may be useful for identifying adults at high-risk for suicide and potentially reducing suicide-related risks at the population level.

\section{Conflict of interest disclosures}

None reported. 
Citation: Jin-Young Min, Kyoung-bok Min (2015) Suicide Behaviors and Health-Related Quality of Life: Results from the Korean Community Health Survey of 393,073 Adults. J Psychiatry 18: 214. doi:10.4172/2378-5756.1000214

Page 7 of 7

\section{Acknowledgement}

This work was supported by the new faculty research fund of Ajou University School of Medicine (Grant nos. M-2014-C0460-00162 and M-2013-C0460-00070.

\section{References}

1. World Health Organization (2007) Suicide prevention (SUPRE). Geneva, Switzerland: World Health Organization.

2. Nock MK, Borges G, Bromet EJ, Cha CB, Kessler RC, et al. (2008) Suicide and suicidal behavior. Epidemiol Rev 30: 133-154.

3. Hawton K, van Heeringen K (2009) Suicide. Lancet 373: 1372-1381.

4. Oquendo MA, Currier D, Mann JJ (2006) Prospective studies of suicidal behavior in major depressive and bipolar disorders: what is the evidence for predictive risk factors? ActaPsychiatrScand 114: 151-158.

5. Cheung K, Oemar M, Oppe M, Rabin R, EuroQol group. EQ-5D User Guide: basic information on how to use the EQ-5D, v2. Available: http:// www.euroqol.org/fileadmin/user_upload/Documenten/PDF/ Folders_Flyers/UserGuide_EQ-5D-5L_v2.0_October_2013.pdf (Accessed 2014 October 20).

6. Goldney RD, Fisher LJ, Wilson DH, Cheok F (2001) Suicidal ideation and health-related quality of life in the community. Med J Aust 175: 546-549.

7. Pompili M, Lester D, Innamorati M, De Pisa E, Amore M, et al. (2009) Quality of life and suicide risk in patients with diabetes mellitus. Psychosomatics 50: 16-23.

8. Kim JH, Kwon JW (2012) The impact of health-related quality of life on suicidal ideation and suicide attempts among Korean older adults. J GerontolNurs 38: 48-59.

9. de Abreu LN, Nery FG, Harkavy-Friedman JM, de Almeida KM, Gomes BC, et al. (2012) Suicide attempts are associated with worse quality of life in patients with bipolar disorder type I. Compr Psychiatry 53: 125-129.

10. Ponizovsky AM, Grinshpoon A, Levav I, Ritsner MS (2003) Life satisfaction and suicidal attempts among persons with schizophrenia. Compr Psychiatry 44: 442-447.

11. Kao YC, Liu YP, Cheng TH, Chou MK (2012) Subjective quality of life and suicidal behavior among Taiwanese schizophrenia patients. Soc Psychiatry PsychiatrEpidemiol 47: 523-532.

12. Yan F, Xiang YT, Hou YZ, Ungvari GS, Dixon LB, et al. (2013) Suicide attempt and suicidal ideation and their associations with demographic and clinical correlates and quality of life in Chinese schizophrenia patients. Soc Psychiatry PsychiatrEpidemiol 48: 447-454.

13. Korean Statistical Information Service. KOSIS data for causes of death (1983-2008) Seoul: Korean Statistical Information Service. 2009.
14. Organization for Economic Co-operation and Development. OECD health data 2013. http://www.oecd.org/els/health-systems/Health-at-aGlance-2013.pdf.

15. Korea Centers for Disease Control and Prevention. Korea Community Health Survey. http://chs.cdc.go.kr/chs/index.do (Accessed 2014 October 20).

16. The EuroQol Group (1990) EuroQol--a new facility for the measurement of health-related quality of life. Health Policy 16:199-208.

17. Nam HS, Kim KY, Kwon SS, Koh KW, Poul K (2007) EQ-5D Korean valuation study using time trade of method. Seoul: Centers for Disease Control and Prevention.

18. Nordström P, Samuelsson M, Asberg M (1995) Survival analysis of suicide risk after attempted suicide. ActaPsychiatrScand 91: 336-340.

19. Kuo WH, Gallo JJ, Tien AY (2001) Incidence of suicide ideation and attempts in adults: the 13-year follow-up of a community sample in Baltimore, Maryland. Psychol Med 31: 1181-1191.

20. Brown GK, Beck AT, Steer RA, Grisham JR (2000) Risk factors for suicide in psychiatric outpatients: a 20 -year prospective study. J Consult ClinPsychol 68: 371-377.

21. Hunt SM (1997) The problem of quality of life. Qual Life Res 6: 205-212.

22. Rogerson RJ (1995) Environmental and health-related quality of life: conceptual and methodological similarities. SocSci Med 41: 1373-1382.

23. Weintraub WS, Spertus JA, Kolm P, Maron DJ, Zhang Z, et al. (2008) Effect of PCI on quality of life in patients with stable coronary disease. N Engl J Med 359: 677-687.

24. Abdel-Kader K, Unruh ML, Weisbord SD (2009) Symptom burden, depression, and quality of life in chronic and end-stage kidney disease. Clin J Am SocNephrol 4: 1057-1064.

25. Khanna D, Maranian P, Palta M, Kaplan RM, Hays RD, et al. (2011) Health-related quality of life in adults reporting arthritis: analysis from the National Health Measurement Study. Qual Life Res 20: 1131-1140.

26. Sullivan PW, Ghushchyan V (2006) Preference-Based EQ-5D index scores for chronic conditions in the United States. Med Decis Making 26: 410-420.

27. Lubetkin EI, Jia H, Franks P, Gold MR (2005) Relationship among sociodemographic factors, clinical conditions, and health-related quality of life: examining the EQ-5D in the U.S. general population. Qual Life Res 14: 2187-2196.

28. Apter AJ, Reisine ST, Affleck G, Barrows E, ZuWallack RL (1999) The influence of demographic and socioeconomic factors on health-related quality of life in asthma. J Allergy ClinImmunol 103: 72-78.

29. Rabin R, de Charro F (2001) EQ-5D: a measure of health status from the EuroQol Group. Ann Med 33: 337-343. 